Paediatr Paedolog 2016 • [Suppl 1]: 51:S10-S15 DOI 10.1007/s00608-016-0380-x Online publiziert: 28. Juni 2016

(c) The Author(s) 2016. This article is available at SpringerLink with Open Access.

CrossMark

Viele Kinder und Jugendliche leiden an chronischen Erkrankungen, wie Rheuma, Diabetes (Typ I), neurologischen Erkrankungen, Stoffwechselerkrankungen oder psychiatrischen Erkrankungen, die einen chronischen, über das Jugendalter hinausgehenden Verlaufaufweisen. Nach verschiedenen Studien wird die Häufigkeit chronischer Erkrankungen bei unter 6-Jährigen mit $9 \%$ angegeben, bei 12 - bis 17-Jährigen mit $17 \%$. Bedingt durch die zunehmend bessere Versorgung erleben über $90 \%$ der Kinder und Jugendlichen mit chronischen Erkrankungen das Erwachsenenalter [1]. Die Studie zur Gesundheit von Kindern und Jugendlichen in Deutschland (KIGGS-Studie) hat erhoben, dass etwa $40 \%$ der Kinder an chronischen Erkrankungen leiden und $14 \%$ der unter 18-Jährigen einen besonderen Bedarf an Gesundheitsversorgung haben [2]. Daher muss - parallel zur psychosozialen und persönlichen Entwicklung - auch die medizinische Betreuung die entsprechenden Angebote liefern, damit das Erwachsenwerden im Allgemeinen und hinsichtlich des Krankheitsmanagements im Speziellen erfolgreich verlaufen kann.

Der Begriff Transition, der für diese Übergänge von der Kinder- und Jugendmedizin in die Erwachsenenmedizin benutzt wird, kann ganzallgemein mit Übergang übersetzt werden und wird als ein multidimensionaler Prozess verstanden, der die Änderungen im Leben einer Person beschreibt [3]. Änderungen verlangen Anpassungsreaktionen und erfordern dadurch das Entstehen neuer Verhaltensweisen und die Integration eines

\title{
L. Thun-Hohenstein
}

Universitätsklinik für Kinder- und Jugendpsychiatrie, Salzburger Landeskliniken/Paracelsus Medizinische Privatuniversität, Christian Dopplerklinik, Salzburg, Österreich

\section{Transitionsmedizin}

\author{
Übergang von kinder- und \\ jugendmedizinischer in \\ erwachsenenmedizinische Betreuung
}

neuen Selbstverständnisses. Als solcher ist Transition ein Teil der normalen gesunden Entwicklung über die gesamte Lebensspanne. All diesen verschiedenen Entwicklungsphasen gemeinsam, sozusagen die zentrale Aufgabe der Entwicklung bildend, ist die Suche nach der persönlichen Identität [4].

\section{》) Transition ist Teil der normalen gesunden Entwicklung über die gesamte Lebensspanne}

Van Gennep ([5]; • Abb. 1) hat in seinem berühmten Buch „Rites de Passage“ die Initiationsrituale traditioneller Gesellschaften in Afrika untersucht. Dabei beobachtete er spezifische Rituale am Übergang in die Erwachsenengruppe. Sie dienten dazu, den Übergang aus der Kinderwelt in die Erwachsenenwelt zu symbolisieren und mit spirituellen und realen Lebenserfahrungen anzuregen und zu bereichern. Dieser Übergangsprozess hat 3 wesentliche Phasen:

Die 1. Phase ist die Trennungsphase. Hierbei kommt es zum Abschied aus der Familie, Abschied aus der Kind- bzw. Jugendlichenposition und zum Hinausgehen in die Welt. Die 2. Phase nennt sich Neuordnungsphase. In dieser Phase ist die Zeit des Übens gekommen, es wird experimentiert. Im Wesentlichen geht es darum, die eigene Identität zu finden; in den Urgesellschaften ging es darum, sein Totem oder sein Lebenssymbol zu finden, um mit dieser Identifikation dann zurückzukehren und in die 3. Phase einzutreten, in die Wiedereingliederungspha- se. Diese bedeutet dann die ritualisierte Aufnahme in die Welt der Erwachsenen.

\section{„Emerging adulthood"}

Das Verständnis der Adoleszenz und des Übergangs zum Erwachsenenalter hat sich in den letzten Jahren dramatisch gewandelt. Die Dauer des Erwachsenwerdens hat sich ausgedehnt und ist deutlich bunter geworden. Arnett beschrieb die Periode zwischen 18 und 25 respektive 30 Jahren als „emerging adulthood“ auftauchendes Erwachsenenalter. Diese Periode ist dadurch gekennzeichnet, dass die Menschen keine Jugendlichen mehr, aber auch noch nicht erwachsen sind. Sie wohnen länger zu Hause, heiraten sehr viel später (etwa mit 29 Jahren), erwerben mehrere Berufsbefähigungen, sind auf der Suche nach dem richtigen Job, der richtigen Lebenssituation. Hauptthemen dieser neuen Entwicklungsphase sind die intensive Exploration der Welt und ihrer Möglichkeiten und Gefahren, eine nach wie vor hohe Instabilität und eine sehr starke Selbstfokussierung. Diese entwicklungspsychologischen Erkenntnisse sind neben den schon bekannten Entwicklungsaufgaben des Adoleszentenalters in dem Prozess der Transition vom ersten Moment an zu berücksichtigen [6]. Insbesondere sind die aus der Instabilität resultierenden Belastungen und das damit verbundene erhöhte Risiko psychischer Erkrankungen zu berücksichtigen. 


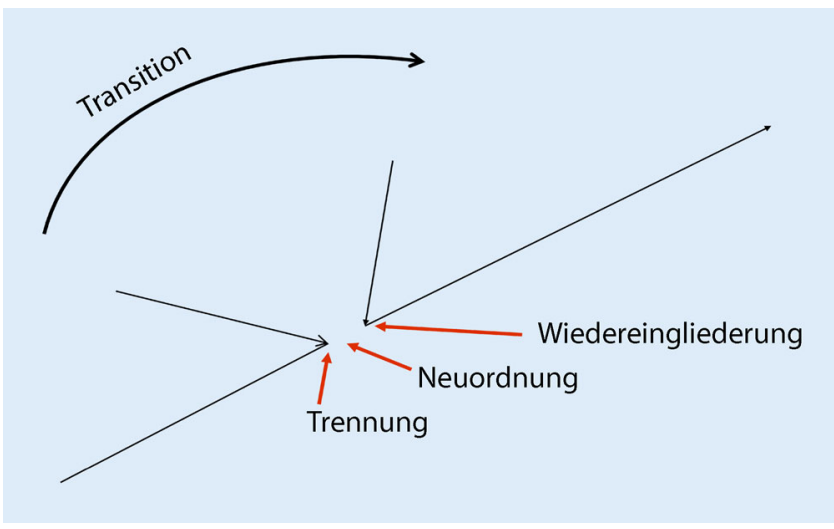

mit einer entsprechenden Begleitung des jungen Menschen. In den meisten Empfehlungen wird eine Kombination von sequenziellem, entwicklungsorientiertem und professionellem Modell angegeben $[1,9,14,15]$.

\section{Einflussfaktoren}

Abb. $1 \triangleleft$ Van Gennepsches Modell der "rites de passage", der Übergangsriten

\section{Transition in der Medizin}

Es handelt sich dabei um einen gezielten und geplanten Prozess von einem kindzentrierten zu einem erwachsenenorientierten Gesundheitssystem, respektive Gesundheitsversorgung $[3,7,8]$.

\section{Ziele}

Das Hauptziel einer gelungenen Transition ist die kontinuierliche medizinische Betreuung ins Erwachsenenalter unter Berücksichtigung der Entwicklung des Jugendlichen $[1,8]$. Es geht dabei um den Aufbau von Versorgungsstrukturen mit möglichst hoher Qualität und möglichst ununterbrochener Versorgung und einer hohen Patientenzentrierung. Gleichzeitig soll die Versorgung alters- und entwicklungsgerecht, kulturell kompetent, flexibel und nachvollziehbar durchgeführt werden [9]. Im Rahmen dieses medizinischen Transitionsprozesses geht es im Sinn der „emerging adulthood“ um den altersentsprechenden Erwerb kommunikativer Fähigkeiten, der Fähigkeit Entscheidungen treffen zu können und sie durchzusetzen. Es geht um die Entwicklung von Selbstfürsorge, Selbstbestimmung und Interessenvertretung [10]. Im spezifischen medizinischen Versorgungssinn geht es um die Stärkung der eigenen Fähigkeit, Symptome und Gesundheitsprozesse zu kontrollieren, und dies möglichst unabhängig von der Gesundheitsversorgung bzw. in einer guten partnerschaftlichen Beziehung mit dieser. Das gemeinsame Ziel dieser Gesundheitsvorsorge und der persönlichen Entwicklung ist das Maximieren von lebenslangen Funktionen und dem den Jugendlichen inneliegenden Entwicklungspotenzial. Äußerst wichtig ist in dieser Zeit auch die Unterstützung der Eltern, da diese einerseits altersbedingt und rechtlich noch Garantenstatus haben, gleichzeitig aber die eigene Entwicklungsaufgabe der Verantwortungsübergabe an den Heranwachsenden zu leisten haben, in einer Phase, wo oft eine erhöhte Kontrolle und Aufmerksamkeit auf die Gesundheitsversorgung wichtig erscheint [11].

\section{Transitionsmodelle}

Es finden sich unterschiedliche Modelle von Transition [12, 13]. Ein Modell umfasst den Schritt aus der Kindermedizin in die Erwachsenenmedizin ohne vorherige Kommunikation und ohne Informationstransfer, die sog. direkte Transition. Ein weiteres Modell ist ein Mehrschrittmodell von der Kindermedizin in eine Transitionsklinik zur Adoleszentenstation und schließlich in die Erwachsenenmedizin; dieses Modell wird als sequenzielle Transition bezeichnet. Ein drittes Modell beschreibt ein als entwicklungsangepasste Transition bezeichnetes Vorgehen, bei dem es vornehmlich darum geht, Skills-Training für den Adoleszenten bzw. entsprechende Unterstützungssysteme aufzubauen, die einen möglichst selbstständigen Weg des Adoleszenten in die Erwachsenenmedizin ermöglichen. Schlussendlich wird die professionelle Transition beschrieben. Hier geht es um eine geplante und gezielte Zusammenarbeit zwischen Kinder- und Jugendmedizin und Erwachsenenmedizin mit einem entsprechenden Wissensund Informationstransfer, gleichzeitig
Der Transitionsprozess wird von vielerlei Faktoren beeinflusst. Haupteinflussgröße ist die Grunderkrankung und deren Status respektive Schweregrad und die konsekutive Beeinträchtigung der Lebensqualität des Jugendlichen [16, 17]. Vonseiten des Adoleszenten hängt dieser Prozess von dessen kognitiven Fähigkeiten, der Wahrnehmung und Selbsteinschätzung der eigenen Erkrankung, von emotionalen und psychischen Faktoren sowie von seinem Wissensstand bezüglich seiner Erkrankung ab. Wesentlichen Einfluss nehmen das Familiensystem und der Einfluss der Gruppe der Gleichaltrigen. Den Transitionsprozess mitbeeinflussend sind die Faktoren des Settings und der Kommunikation: das gewählte Transitionsmodell, die Beziehung des Adoleszenten zum Behandlungsteam, die Komplexität der Erkrankung und Behandlung und v. a. die Interferenz der Behandlungsmodalitäten mit den Bedürfnissen des Adoleszenten und seines Lebensstils [11].

\section{I) Die Erwachsenenmedizin ist am Patienten orientiert im Sinn einer Beratungs- und Empfehlungsmedizin}

Aufgrund dieser multifaktoriellen Gemengelage kann es auch zu ausgeprägten Problemen in diesem Transitionsprozess kommen. Faktoren des Adoleszenten (Kognition, Persönlichkeitsstrukturen, Krankheitsverständnis, psychologische Faktoren etc.), Faktoren der Umgebung (Familie, Peer-Gruppe) und Faktoren des Settings, wie unklare Kommunikation, schlechte Organisation des Transitionsprozesses, schwere und komplexe Erkrankungsbilder, fehlende Ressourcen, geographische und strukturelle Gegebenheiten, sowie fehlende flächen- 
deckende Versorgung stellen große Probleme dar [11].

Ganz wesentlich ist aber auch der Paradigmenwechsel zwischen Jugendmedizin und Erwachsenenmedizin. Die Jugendmedizin sieht sich eher fürsorglich paternalistisch, an der Familie orientiert und ganzheitlich versorgend. Sie ist prinzipiell interdisziplinär, aber auch zentralisiert organisiert und sieht sich als vernetzter Generalist. Ganz wesentlich ist auch die Entwicklungsorientierung, die Orientierung am psychosozialen Umfeld und an der Prävention. Die Erwachsenenmedizin hingegen ist eher am Patienten orientiert im Sinn einer Beratungs- und Empfehlungsmedizin, die Patientenautonomie wird prinzipiell akzeptiert und respektiert. In der Regel ist Erwachsenenmedizin monodisziplinär, eher dezentralisierend, wird von Hausärzten und verschiedenen Spezialisten übernommen. Im Gegensatz zur Jugendmedizin ist die Erwachsenenmedizin i. d. R. eher problemfokussiert und agiert weniger im Sinn der ganzheitlichen Versorgung des betroffenen Menschen [15].

\section{Wie muss Transition organisiert sein?}

McDonagh und Viner [10] beschreiben Schlüsselelemente transitionaler Betreuung, die unabdingbar vorhanden sein müssen. Die gesamte Transition sollte rechtzeitig bzw. früh mit einer zentralen Ansprechperson, die für den Prozess verantwortlich ist, beginnen. Ideal ist das Vorhandensein schriftlicher Vereinbarungen zwischen der Kinder- und Jugendmedizin und der Erwachsenenmedizin der jeweiligen Fachrichtung, in der die Aufgaben, Strukturen und Voraussetzungen für die Transition genau definiert sind und in Prozessen niedergelegt sind. In Bezug auf den einzelnen Patienten geht es darum, sich an dessen Entwicklungsschritte und Entwicklungsmöglichkeiten anzupassen, flexibel zeitlich zu planen und mithilfe von Skills-Training Kommunikation, Entscheidungsfindung, Problemlösungsverhalten und Selbstfürsorge zu verbessern, sodass eine erwachsene selbstbestimmte Übernahme der Gesundheitsversorgung

Paediatr Paedolog 2016 • [Suppl 1]: 51:S10-S15 DOI 10.1007/s00608-016-0380-x

(c) The Author(s) 2016. This article is available at SpringerLink with Open Access.

\section{Thun-Hohenstein}

Transitionsmedizin. Übergang von kinder- und jugendmedizinischer in erwachsenenmedizinische Betreuung

\section{Zusammenfassung}

Transitionsmedizin ist die medizinische Versorgung jugendlicher Patienten an der Schwelle zum Erwachsenwerden. Dieser Prozess ist definiert als gezielter und geplanter Prozess von einem kindzentrierten zu einem erwachsenenorientierten Gesundheitssystem. Dabei sind die verschiedenen Kulturen der Kinder- und Jugendmedizin und der Erwachsenenmedizin, der Entwicklungsaufgaben und deren Bewältigung durch den Jugendlichen sowie Umfeldfaktoren zu berücksichtigen. Die Qualität der Transition ist einerseits von kulturellen und strukturellen Faktoren abhängig (Kooperation zwischen den Disziplinen, strukturierter
Transitionsplan), vom genauen Assessment des Jugendlichen in all seinen psychosozialen, kognitiv-emotionalen und medizinischen Qualitäten. Je besser die Struktur, je besser geplant die Transition ist, umso erfolgreicher wird sie sein und damit den Gesundheitsprozess und die Lebensqualität des Adoleszenten verbessern.

\section{Schlüsselwörter}

Bereitstellung der Gesundheitsversorgung . Interdisziplinäres Gesundheitsteam . Medizinische Ausbildung · Leitlinien zur Gesundheitsplanung $\cdot$ Kontinuität der Patientenbetreuung

\section{Transition medicine. Transfer from paediatric and adolescent to adult medical care}

Abstract

Transition medicine is the medical care of young patients on the edge to adulthood. It is defined as a specific, planned process from a child-centred to an adult-orientated health care system. Therefore, the various cultures of paediatric and adolescent medicine and of adult medicine, of development challenges and how young people overcome them as well as environmental factors should be taken into consideration. For one thing, the quality of transition is dependent on cultural and structural factors (cooperation between the disciplines, structured transition plan), of precise assessment of the young person with all of his or her psychosocial, cognitive/emotional and medical characteristics. The better the structure, the better planned the transition, the more successful it will be and with it the health process and quality of life of the adolescent.

\section{Keywords}

Delivery of health care - Interdisciplinary health team - Medical education - Health planning guidelines - Continuity of patient care durch den jungen Menschen möglich ist. Es empfiehlt sich, einen schriftlichen Transitionsplan bereits im Alter von 12 Jahren gemeinsam mit Jugendlichen und Familie zu erstellen und diesen mit der Erwachsenenmedizin entsprechend abzustimmen. Dabei werden entsprechende administrative Organisation und Unterstützung, reguläre Überprüfung dieser Vereinbarungen und entsprechende Updates benötigt. Ganz wichtig ist in diesem Prozess auch die Involvierung der Hausärzte, die über den Prozess zumindest regelmäßig informiert werden müssen, wenn sie nicht selbst aktiv planend und beteiligt in diesem Prozess mitwirken [10].

\section{Wie sieht ein gelingendes Transitionsmodell aus?}

Prinzipiell gilt es, feste interdisziplinäre Strukturen aufzubauen, die bestimmte Standards der Transition erfüllen und kontinuierlich gewährleisten. Grundlegend ist das Erarbeiten eines Statuts („policy“), das den Transitionsprozess genau beschreibt und die Verantwortlichkeiten festlegt. Administrative und kooperative Strukturen müssen geschaffen und die Finanzierung derselben gesichert werden [8]. Die American Academy of Pediatrics [9] hat in ihrem Konsensusstatement einen Algorithmus erarbeitet. Der erste Schritt beinhaltet die Aktivierung des 


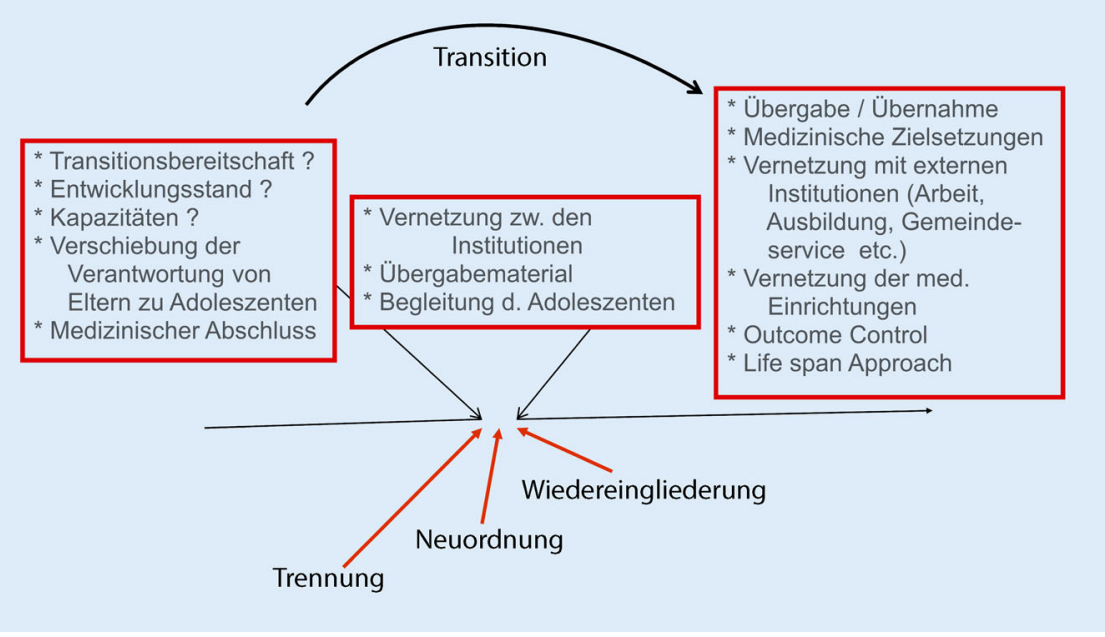

Abb. $2 \Delta$ Idealtypische Transition in der Medizin anhand des Van-Gennepschen-Modells

Transitionsprozesses im Alter ab 12 Jahren. Die Jugendlichen werden hinsichtlich ihrer Erkrankung und deren Versorgung genau untersucht und es wird gemeinsam mit ihnen erarbeitet, wie viel und welche Unterstützung sie während des Transitionsprozesses benötigen werden. Die psychosozialen Fertigkeiten und Fähigkeiten werden überprüft, das Vorliegen von speziellen Bedürfnissen erhoben und - wenn notwendig - einer Betreuung zugeführt. Im Alter von 16 bis 17 Jahren sollte der Transitionsplan ausgearbeitet vorliegen und mit dem Jugendlichen, allen Experten und den Eltern abgestimmt sein [11]. Mit 18 Jahren sollte der Übergang umgesetzt und die Betreuung durch die Erwachsenenmedizin übernommen werden. In - Abb. 2 werden die Inhalte des Transitionsprozesses anhand des Van-Gennepschen-Modells übersichtlich dargestellt. Im Rahmen der Trennungsphase gilt es als allererstes, die Transitionsbereitschaft des Jugendlichen und seines Systems festzustellen. Wichtig dabei ist die Feststellung des Entwicklungsstands, der Fertigkeiten und Fähigkeiten im Umgang mit der eigenen Lebenssituation und insbesondere der Erkrankung und deren Behandlung. Es sollen die Kapazitäten der eigenen Versorgung, der Versorgung der Erwachsenenmedizin und aber auch der Eltern und des Adoleszenten geklärt sein und miteinander in eine vernünftige Relation und Abfolge gebracht werden. Es gilt, die Eltern darin zu unterstützen, die Vergung von den Eltern zum Adoleszenten hin $\mathrm{zu}$ verschieben und beide dabei $\mathrm{zu}$ unterstützen, diesen Prozess erfolgreich durchzuführen. Last, but not least geht es darum, einen guten kinder- und jugendmedizinischen Abschluss zu entwickeln unter Berücksichtigung der somatischen Notwendigkeiten, aber auch unter Einbeziehung des gesamten psychosozialen Umfelds und der zukünftigen medizinischen Betreuung.

In der Neuordnungs- oder Übergangsphase geht es darum, sich mit den nachsorgenden Institutionen zu vernetzen, das Übergabematerial vorzubereiten und den Adoleszenten in diesem Prozess zu begleiten.

\section{\) Outcomekontrollen müssen festgelegt werden}

Die Wiedereingliederungsphase beginnt mit der Übergabe bzw. Übernahme der medizinischen Versorgung durch die Erwachsenenmedizin. Es gilt, die neuen medizinischen Zielsetzungen zu definieren, sich mit externen Institutionen, wie Arbeitsplatz, Ausbildung, Gemeindeservice etc., zu vernetzen und auch die medizinischen Partner in der Versorgung entsprechend einzubeziehen. Festzulegen sind Kontrollen des Outcomes. Prinzipiell ist ab diesem Zeitpunkt nicht nur eine punktuelle Unterstützung des jungen Erwachsenen notwendig, sondern antwortung für die Gesundheitsversor- es muss die Betreuung unter dem Aspekt eines „life-span approach“ stehen [13].

Verschiedenste Studien haben nachgewiesen, dass eine Berücksichtigung der verschiedenen Aspekte des beschriebenen Transitionsprozesses zu deutlich besseren Übergaben an die Erwachsenenmedizin führen (s. Übersicht bei [18]) und damit die Prognose der Grundkrankheit wesentlich verbessern. Qualitätsmanagement wird allgemein als ein wesentlicher Beitrag zum Gelingen des Transitionsprozesses angesehen. Dabei geht es um die standardisierte Erhebung und Analyse von Faktoren der Vorbereitung (z. B. Transitionsbereitschaft des Adoleszenten, Lebensqualität vor, während und nach der Transition etc.), der Überprüfung der Übergabe und der Nachsorge nach der Transition (Gesundheitsversorgung, Lebensqualität und krankheitsspezifisches Outcome; [1]).

\section{Korrespondenzadresse}

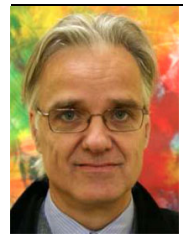
Prof. Dr. L. Thun-
Hohenstein
Universitätsklinik für Kinder- und Jugendpsychiatrie, Salzburger Landeskliniken/ Paracelsus Medizinische Privatuniversität, Christian Dopplerklinik Ignaz Harrerstraße 79, 5020 Salzburg, Österreich L.Thun-Hohenstein@salk.at

Prof. Dr. L. Thun-Hohenstein Facharzt für Kinderund Jugendheilkunde, Facharzt für Kinder- und Jugendpsychiatrie, Zusatzfacharzt für Neuropädiatrie; seit 2009 Vorstand der Univ.-Klinik für Kinder- und Jugendpsychiatrie Salzburg; Leiter des Kinder- und Jugendpsychiatrischen Diensts „Kinderseelenhilfe“, ProMente, Salzburg; Präsident der Österr. Gesellschaft für Kinder- und Jugendpsychiatrie (2006-2008, Präpräsident 2004-2006, Pastpräsident 2008-2012). Lebenslauf: 1955 Geburt in Wien; 1973 Matura Gymnasium Kremsmünster, OÖ; 1979 Promotion zum Dr. med. univ., Universität Wien; 1986 Facharzt für Kinder- und Jugendheilkunde; 1988 Studienaufenthalt UK für Kinder- und Jugendheilkunde, Abteilung für Wachstum und Entwicklung, Zürich; 1991/92 Oberarzt, Abteilung Neuropädiatrie, UK für Kinder- und Jugendheilkunde, Zürich; 1993 Zusatzfacharzt für Kinder- und Jugendneuropsychiatrie; 2007 Privatdozent für Kinder-und Jugendpsychiatrie; 2009 Berufung zum Professor für Kinder- und Jugendpsychiatrie; 2012 Berufung zum Längskoordinator Soziale Kompetenz im Medizinstudium an der Paracelsus Medizinischen Privatuniversität. Arbeits- und Forschungsgebiete: Allgemeine Kinder- und Jugendpsychiatrie, Evaluation und Qualitätsmanagement, frühe Kindheit, Entwicklung, Versorgung, Epidemiologie, Gewalt am Kind, Essstörung, Lehre Soziale Kompetenz 


\section{Einhaltung ethischer Richtlinien}

Interessenkonflikt. L. Thun-Hohenstein gibt an, dass kein Interessenkonflikt besteht.

Dieser Beitrag beinhaltet keine vom Autor durchgeführten Studien an Menschen oder Tieren.

Open Access. This article is distributed under the terms of the Creative Commons Attribution 4.0 International License (http://creativecommons.org/ licenses/by/4.0/), which permits unrestricted use, distribution, and reproduction in any medium, provided you give appropriate credit to the original author(s) and the source, provide a link to the Creative Commons license, and indicate if changes were made.

\section{Literatur}

1. Sharma N, O'Hare K, Antonelli RC, SawickiGS(2014) Transition care: future directions in education, health policy, and outcomes research. Acad Pediatr 14(2):120-127

2. Scheidt-Nave C, Ellert U, Thyen U, Schlaud M (2007) Prävalenz und Charakteristika von Kindern und Jugendlichen mit speziellem Versorgungsbedarf im Kinder+Jugendgesundheitssurvey (KIGGS) in Deutschland. Bundesgesundheitsblatt Gesundheitsforschung Gesundheitsschutz 50:750-756

3. Meleis AL (2007) Theoretical nursing: development and progress. Lippincott, Williams and Wilkins, Philadelphia

4. Seiffge-Krenke I (2014) Identität im Wandel und therapeutische Herausforderung. Forum Psychoanal 30(1):85-108

5. Gennep A van (1960) The rites of passage. University of Chicago Press, Chicago

6. Seiffge-Krenke I (2015) „Emerging Adulthood“ Forschungsbefunde zu objektiven Markern, Entwicklungsaufgaben und Entwicklungsrisiken. ZPsychiatr Psychol Psychother 63(3):165-173

7. Blum RWM, Garell D, Hodgman $C H$, Jorissen TW, Okinow NA, Orr DP, Slap GB (1993) Transition from child-centered to adult-health care systems for adolescents witch chronic conditions. A position paper of the Society for Adolescent Medicine. J Adolesc Health 14:570-576

8. Blum RWM, Hirsch D, Kastner TA, Quint RD, Sandler AD (2002) A consensus statment on health care transitions for young adults with special health care needs. Pediatrics 110:1304-1306

9. Cooley WC, Sagerman PJ (2011) Supporting the health care transition from adolescence to adulthood in the medical home. Pediatrics 128:182-200

10. McDonagh JE, Viner R (2006) Lost in transition? Between pediatric an adult services. BMJ 332:435-436

11. Michaud PA, Suris J-C, Viner R (2004) The adolescent with a chronic condition. Part II: healthcare provision. Arch Dis Child 89:943-949

12. McDonagh JE (2005) Growing up and moving on: transition from pediatric to adult care. Pediatr Transplant 9:364-372

13. Reincke M, Lehnert $H$ (2009) Transition in der Inneren Medizin. Internist 50:1189-1193

14. McManus MA, White $P$, Barbour A, Downing B, Hawkins K, Quion N, Tuchman LK, Cooley WC, McAllister JW (2015) Pediatric to adult transition: a quality improvement model for primary care. JAdolesc Health 56:73-78
15. Staa AL van, Jedeloo S, Meerten J van, Latour JM (2011) Crossing the transition chasm: experiences and recommendations for improving transitional care of young adults, parents and providers. Child Health Care Dev 37(6):821-832

16. McManus MA, PollackLR, Cooley WC, McAllister JW, Lotstein D, Strickland B, Mann MY (2013) Current status of transition preparation among youth with special needs in the United States. Pediatrics 131:290-297

17. Kelly AM, Kratz B, Bielski M, Mann-Rinehart $P$ (2002) Implementing transitions for youth with complex medical conditions using the medical home model. Pediatrics 110:1322-1327

18. Crowley R, Wolfe I, Lock K, McKee M (2011) Improving the transition between pediatric and adult health care: a systematic review. Arch Dis Child 96:548-553 
Hier steht eine Anzeige.

Springer
Hier steht eine Anzeige.

글 Springer 